\title{
Life Review Using a Life Metaphoric Game to Promote Intergenerational Communication
}

\author{
SEYEON LEE, Graduate School of Culture Technology, KAIST, Republic of Korea \\ HYUNYOUNG OH, Graduate School of Culture Technology, KAIST, Republic of Korea \\ CHUNG-KON SHI, Graduate School of Culture Technology, KAIST, Republic of Korea \\ YOUNG YIM DOH ${ }^{*}$, Graduate School of Culture Technology, KAIST, Republic of Korea
}

This study explores whether a life metaphoric game can effectively facilitate communication between senior participants and young adult partners during a life review activity. Life review provides older adults with opportunities to organize their past experiences, rediscover the meaning of life, and prepare for their future life and eventual death. We held workshops in which 33 senior participants (ages 51-85) co-played a commercial game, titled "Long Journey of Life," paired with young adult partners (ages 19-23). The young partners asked senior participants about their associated memories during the life review activity. Through inductive thematic analysis, we found seven communication themes during the life review activity: reminiscence, future and death preparation, life advice, appreciation and evaluation, small talks involving self-disclosure, interpretation of metaphors, and explanation of game control methods. We investigated the relationships between communication themes and game design elements that promote conversation. In addition, we identified how participants' responses to the game differ depending on the player's characteristics and generation. Game design suggestions for an effective intergenerational life review using digital games were offered.

CCS Concepts: • Human-centered computing $\rightarrow$ Empirical studies in collaborative and social computing.

Additional Key Words and Phrases: Digital Games; Intergenerational Play; Co-playing; Life Review; Reminiscence; Older Adults

ACM Reference Format:

Seyeon Lee, Hyunyoung Oh, Chung-Kon Shi, and Young Yim Doh. 2020. Life Review Using a Life Metaphoric Game to Promote Intergenerational Communication. Proc. ACM Hum.-Comput. Interact. 4, CSCW2, Article 98 (October 2020), 21 pages. https://doi.org/10.1145/3415169

\section{INTRODUCTION}

Issues regarding senior citizens' welfare have drawn considerable attention worldwide, highlighting the need for healthier lifestyles at older ages. With the global problem of an aging population, extensive research is needed to design and implement intergenerational activities to enhance communication and interaction between young and older people [48]. Playing games is a pastime that is often associated with younger generations; however, middle-aged and older adults are

${ }^{*}$ Corresponding Author.

Authors' addresses: Seyeon Lee, birdkite@kaist.ac.kr, Graduate School of Culture Technology, KAIST, Republic of Korea, 291 Daehak-ro, Yuseong-gu, Daejeon, 34141; Hyunyoung Oh, microtrombo@kaist.ac.kr, Graduate School of Culture Technology, KAIST, Republic of Korea; Chung-Kon Shi, chungkon@kaist.ac.kr, Graduate School of Culture Technology, KAIST, Republic of Korea; Young Yim Doh, yydoh@kaist.ac.kr, Graduate School of Culture Technology, KAIST, Republic of Korea.

Permission to make digital or hard copies of part or all of this work for personal or classroom use is granted without fee provided that copies are not made or distributed for profit or commercial advantage and that copies bear this notice and the full citation on the first page. Copyrights for third-party components of this work must be honored. For all other uses, contact the owner/author(s).

(C) 2020 Copyright held by the owner/author(s).

2573-0142/2020/10-ART98

https://doi.org/10.1145/3415169

Proc. ACM Hum.-Comput. Interact., Vol. 4, No. CSCW2, Article 98. Publication date: October 2020. 
also familiar with games [20], many of them having played games since their youth [45]. Digital games are associated with well-being for older players [3] and can provide cognitive and physical benefits [32]. Meanwhile, life review is a proven intervention method that positively influences the mental health of older adults in the field of psychology [29, 53]. Traditional life reviews were conducted mainly as interviews between speakers and listeners [31]. Previous studies have explored intergenerational life review activities $[15,61]$ and intergenerational game-playing activities [16, $18,44,60]$ separately, but few have combined the two activities. In this study, we propose digital games as a tool to help recall past memories and create an enjoyable atmosphere between the senior generation speaker and the young generation listener. We designed and conducted intergenerational life review activities using a digital game. By analyzing conversations between senior citizens and young partners, we explored whether digital games can be a medium that can promote communication during intergenerational life review activity.

\subsection{Reminiscence and Life Review of Older Adults}

Reminiscence refers to recalling memories of past events, either individually or in a group. Life review is one of the most important aspects of reminiscence, in which people organize their life events in chronological order with more structured themes [8, 9, 29, 54]. Three functions of reminiscence have been classified [53]: a social function that enhances bonding by sharing private memories with others; an instrumental function that recalls past experiences and helps people confront present difficulties; and an integrative function that creates identity by connecting to the future. Reminiscence has also been shown to promote the ability to memorize specific events, encourage communication and social activities in patients with dementia, and enhance older adults' socioemotional well-being by reducing depression and loneliness [14, 29, 49, 53] Furthermore, successful reminiscence and life review can promote a sense of ego-integrity [53] Erikson identified "ego-integrity" as the final stage of psychosocial development [24]. Ego-integrity is a sense of coherence and wholeness that helps people accept their lifecycle as something that was inevitable. People can organize their lives by resolving past conflicts and assigning value to them accordingly.

In traditional life reviews, listeners ask questions and reviewers provide answers. Various intervention programs have been developed to help reviewers recall more memories during this process $[34,53]$. Haight suggested eight forms of life review as an extension of the model proposed by Erikson [30, 31]. In these studies, the role of the listeners in facilitating successful life review was especially highlighted as they could recognize the reviewers' characteristics and apply effective strategies accordingly. Recent studies have also emphasized the necessity of a dyadic approach in the intervention process, which is particularly helpful in promoting communication between the listener and reviewer, improving the quality of their relationship, and enhancing their mutual enjoyment of the process. Intergenerational life review in particular can benefit both generations [34], allowing the older partner to revive a storytelling role and the younger partner to acquire knowledge of living history and wisdom from cultural legacy [29].

\subsection{Use of Technology and Gamification for Reminiscence}

Existing reminiscence interventions have applied technology and digital systems. For example, multimedia such as photos, videos, and music have been used to promote reminiscence. More recently, specially developed applications have allowed users to interact with devices such as computers, tablet personal computers (PCs), and virtual reality (VR) headsets. Four main benefits of applying technology for reminiscence therapy have been suggested [37]. First, technology can allow for greater participation by mitigating motor and sensory impairments, and compensating for memory deficits. Second, technology can take advantage of longer lasting abilities, such as sensory

Proc. ACM Hum.-Comput. Interact., Vol. 4, No. CSCW2, Article 98. Publication date: October 2020. 
awareness, musical responsiveness, and emotional memory. Third, technology can alleviate the burden of providing therapy by gathering and organizing both personal and general material, or by enabling remote therapy and tailoring. Finally, technology can be used to evaluate participants' progress and use of the system [37].

Studies have employed digital technologies that store and sort various types of data, such as photos, text, and videos. Applications have been introduced to collect favoured data based on reviews of photos and music, thereby promoting reminiscence $[11,26]$. Tagging systems have been developed to bind personal experiences to physical objects, places, emotions, individuals, etc $[28,35,38,42]$. These applications focus on promoting memory retrieval by managing various types of mementos. Other technologies focus on promoting dyadic relationships between older adult users, families, and caregivers. When older adults with cognitive impairment and their caregivers watched personalized multimedia biographies together, the caregivers were able to better understand their patients and facilitate more social interaction [17]. Another study suggested a photo-based interaction system using social networking services (SNS), through which family members living away from each other can share photos and memories [7].

As part of technological attempts, game or gamification design has also been used to promote reminiscence. The interactivity of game play, which distinguishes video games from other passive media such as movies, can also promote life reflection. Players must make certain "actions" to continue playing a game, and this enables players to reflect upon themselves in terms of their avatar [19]. When people play games, their personality and values are reflected in their in-game behaviour [27]. This interactivity allows players to easily sympathize with game characters and connect their lives to the story in the game.

Several studies have applied games to reminiscence. "Anecdote" applied the process of life review to a board game wherein each stop depicts a theme from four life stages: childhood, adolescence, adulthood, and life in general. Players move by rolling the dice and tell a personal story according to the theme of the stop they land on [1]. The game "Memoir Monopoly" integrates participants' personal materials in the game play using an iPad so that older adults recall memories based on photos, music, and quizzes in the game [13]. The game "What Remains?" applied a persuasive game design that allowed patients with Alzheimer's disease to tell their caregivers stories based on pictures of their personal objects [10]. Gamification has also been applied to promote reminiscence through a design workshop in the Lebensnetz Project [40]. Such examples demonstrate that games can promote communication between older adults and caregivers. Similarly, various game design techniques have been applied to reminiscence interventions to improve participants' cognitive ability and selfesteem. "Memory matters" presented quizzes using pictures and music representing the participants' past [33]. The MINWii Project played familiar melodies to patients with Alzheimer's disease and dementia [5]. "Food stamps" used objects with cultural symbols to enhance memory capacity and calculation ability [12].

Attempts have been made to apply digital games for reminiscence, aiming to provide entertainment with novel ideas and encourage social interaction; however, applications to provide opportunities to integrate life experiences remain largely unexplored. Furthermore, psychological evaluation in the field of new media is limited. Reminiscence techniques must not only evoke memories but also integrate a person's emotions, self-concept, and past experiences in the subconscious [52]. In particular, life review involves consulting techniques that can assign positive meaning to negative experiences [53]. However, few studies have examined the use of games as a tool for life review. 


\subsection{Intergenerational Play for Promoting Communication}

Studies show that co-playing increases the fun of games and players' positive emotions $[25,36,51$, 58]. In particular, these benefits are enhanced when older and younger generations play together $[43,55]$. Motivations for young people playing games with their older family members, such as parents, grandparents, and uncles/aunts, were fun and bonding with family members [57]. Intergenerational play can be helpful for reinforcing family bonds, enhancing reciprocal learning, increasing understanding of other generations, and reducing social anxiety [18]. In addition, it was proved that intergenerational play can develop positive perceptions toward other age group members [16], increase the breadth and depth of self-disclosure, and promote relationship closeness in younger and older adults [44].

However, a gap in technology experience and skills between older adults and young people can cause an imbalance in interaction [2]. When playing intergenerational games, young people take on the role of teaching the game, thus, communication between the elderly and the young is not symmetrical and reciprocal [57]. Zhang and colleagues [60] performed conversation analysis to study how the structure of conversation between older adults and young partners during intergenerational play provides opportunities for situated learning. Older adults adapted to and understood game-playing activities through young partners' guidance. Older adults asked questions and received feedback from young partners to learn the game mechanism. Young partners answered their situated questions, corrected mistakes, focused attention on key concepts, and explained game rules and strategies. The main challenge of intergenerational play for young people was that older family members were unfamiliar with game rules and mechanisms; moreover, skill gaps, different cohort knowledge, and older adults' physical and cognitive decline could be obstacles to intergenerational play [57]. Therefore, more research will be needed to promote reciprocal interaction between the two generations in intergenerational play.

\subsection{Research Objective}

Although life review is a meaningful activity for both generations, it is difficult to practice in daily life. Most listeners who practice life reviews with older adults are staff members, students, or family members with limited prior training. Therefore, they cannot effectively guide the reviewer to rediscover memories and integrate their life experiences. Furthermore, the need to collect and organize mementos to aid the life review process can be a burden [17, 34]. Additionally, even if intergenerational play can enhance close relationships between two generations $[16,18]$, there are limitations because of communication imbalance [57,60].

To overcome these difficulties, we propose intergenerational play of an life metaphoric game as an alternative setting that can promote rich dyadic communication for life review. The present study explored whether digital games could be an effective tool to facilitate intergenerational communication in the life review activity.

Research questions:

- RQ1. What kinds of intergenerational communication themes emerge in life review using a life metaphoric game?

- RQ2. How can the elements of the life metaphoric game promote intergenerational communication?

- RQ3. How do participants' responses to life review using a life metaphoric game differ depending on the player's characteristics and generations?

- RQ4. What should be considered to effectively design a game for intergenerational life review? 


\section{METHODS}

\section{1 “Games for Life Review” Workshop}

2.1.1 Participants. This research analyzed the experience of participants in the "Games for Life Review" workshop, which was part of a larger living lab project called "Intergenerational Play Workshops (IPW)." The "Games for Life Review" was a one-day session that lasted approximately an hour. Thirty-four senior citizens and 11 undergraduate students participated in this workshop. Four classes were formed with six to ten pairs, each comprising one senior participant and one young partner. Due to the imbalance in numbers, young partners participated in all classes and were paired with multiple senior participants. Furthermore, most partners knew each other beforehand as they had met five times and played other games together in the IPW. There were two cases that couples did not know each other because they did not play games in pairs in the previous sessions of the IPW. Ethical approval for this study was obtained from the institutional review board of KAIST. The participants were paid KRW 10,000 (approximately USD 8) as compensation.

We recruited participants for all the activities of the IPW (8-week program), and any participants who were recruited for the IPW could join the "Games for Life Review." Activities involving older adult participants were conducted at a senior welfare center, and activities involving middle-aged adult participants were held at an education center for the $50+$ generation. Both centers were located in the capital area of South Korea.

Participants at the welfare center were recruited offline over one month; two social workers affiliated with the senior welfare center helped to select older adult participants who were mentally and physically healthy. Because we chose games novice players can learn easily, previous game experiences and technological skills using digital devices were not considered as screening criteria. On the other hand, middle-aged participants were recruited online through the education center's homepage. For gaining participation informed consent forms, the purpose and process of the workshops were explained, and those who did not want to participate were excluded. Table 1

Table 1. Demographic distribution of senior participants $(\mathrm{N}=33)$

\begin{tabular}{cll}
\hline Group & Middle-aged adult group (N=14) & Older adult group (N=19) \\
\hline Age & $51-55(3), 56-60(4), 61-65(7)$ & $\begin{array}{l}66-70(5), 71-75(7), 76-80,(5) \\
81-85(2)\end{array}$ \\
Sex & Female (8), Male (6) & Female (16), Male (3) \\
Job Status & Employed (6), & Employed (5), \\
& Retired/Homemaker (8) & Retired/Homemaker (14) \\
Marital Status & Married (13), & Married (15), Widowed (3), \\
& Divorced/Separated (1) & Divorced/Separated (1) \\
Education & Elementary/Middle (1), High (2), & Elementary/Middle (11), High (5), \\
(School) & Undergraduate (8), Graduate (3) & Undergraduate (1), Graduate (2) \\
Household & Low: Less than 2 Million Won (4), & Low: Less than 2 Million Won (12), \\
Monthly & Middle: 2-8 Million Won (7), & Middle: 2-8 Million Won (6), \\
Income & High: More than 8 Million Won (2), & High: More than 8 Million Won (1) \\
& N/A (1) &
\end{tabular}

* Survey was conducted in KRW. 
reports demographic information of the senior participants. One participant was excluded from the data analysis due to a technical problem with audio recording.

In addition, the young partners comprised nine females and two males, with an average age of 21.45 (ages 19-23). We sent an online application link to undergraduate students in the Department of the Senior Industry at Kangnam university, and 11 students volunteered.

We held an orientation session for young partners to provide basic knowledge of reminiscence and life review, the process of the workshop, and proper manners for interaction with middle-aged and older adults. They were instructed to ask questions that facilitate reminiscence during the workshop, but refrain from asking too many questions if senior participants were reluctant to answer. The researchers did not provide a list of specific questions, hence, young partners were encouraged to generate their own questions. After the activity was finished, researchers held a debriefing session that only young partners participated in.

2.1.2 Life Metaphoric Game "Long Journey of Life". A mobile game application called "Long Journey of Life" was selected for use in this study [56]. Developed by PINX studio and launched in 2018, the game uncovers the course of a person's life from birth to death while a boat sails across the ocean. Figure 1 shows game scenes and stories in each stage.

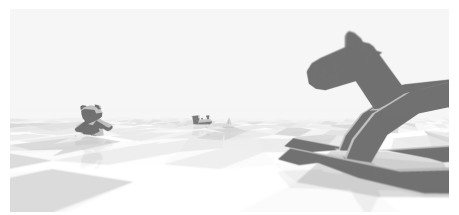

(a) Birth and childhood: A small boat falls from a waterfall. The boat passes the teddy bear and wooden horse.

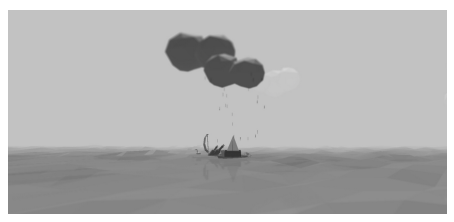

(d) Adulthood: Clouds float above and rain pours. The player boat meets several other boats as they pass by.

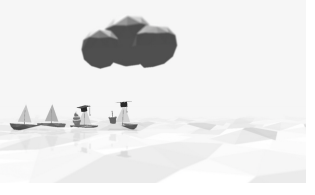

(g) Graduation: The child grows up and graduates from school. The child boat finds its mate and leaves the parents.

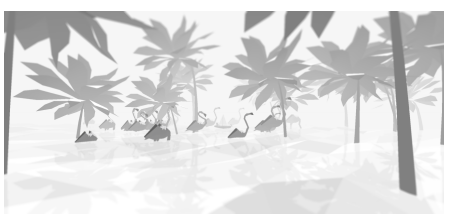

(b) Teenage: The boat passes through a dense forest with cranes.

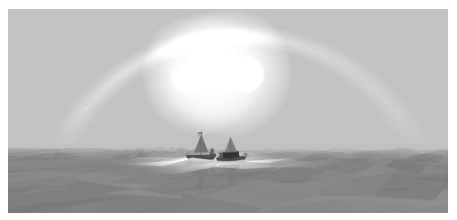

(e) Relationship and marriage: The player boat meets a lover. When the two boats bump into each other, a baby boat is born.

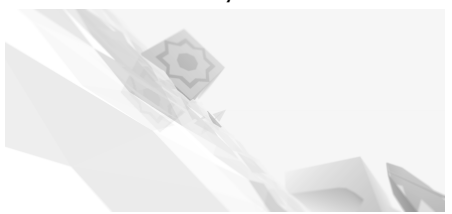

(h) Late age: The spouse boat disappears. The world is turned upside down and the player boat keeps sailing alone.

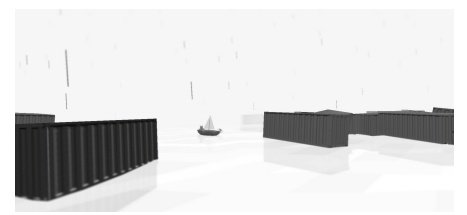

(c) Adolescence: It starts to rain, and container boxes block the way ahead. A whale helps to navigate the container boxes.



(f) Family: Family boats go to the amusement park and ride a merrygo-round.

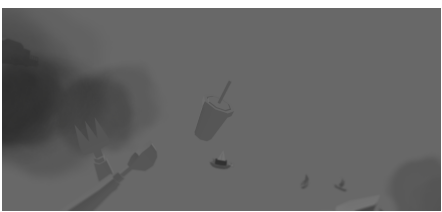

(i) Death: The player boat says goodbye to family, and falls off the waterfall. It encounters everything it has met.

Fig. 1. Screenshots from the game "Long Journey of Life." @PINX 2018. Reuse not permitted. 
Several aspects of the game are of considerable relevance to this study. First, it can support reminiscence as it displays many stages that metaphorize life events in a short playing duration. The game's grayscale visual design and soft music encourage players to contemplate their lives. Finally, the mobile version is easy for senior players to access and control: using two buttons, the character in the game can be moved in only two directions, forward and backward. A simple user interface was preferred because complexity could disrupt intergenerational communication during the life review. While playing this game, senior participants and young partners can stop their actions and talk at any time.

2.1.3 Activity Design of the "Games for Life Review" Workshop. The activity consisted of three steps: co-playing, paired discussion, and focus group interview (FGI). Step 1 (co-playing): Each pair was provided with a 10.1-inch tablet PC for the session. During co-playing "Long Journey of Life" for 20-30 minutes, each pair was asked to share memories of their lives. The young partners helped senior participants by guiding them in game playing and asking questions to promote reminiscence. The researchers provided help when a pair encountered difficulties, but tried to engage minimally otherwise. Step 2 (paired discussion): after playing, participants discussed memories and feelings from each game scene for 15-20 minutes. Young participants filled out a sheet distributed by the researchers for use in the post-game discussions on how senior participants contemplated each stage in the game; the sheet had a table, with different stages of life on each line, and a blank column next to it where the young partners summarized and noted the senior participants' recollection. They also discussed the game's strengths, weaknesses, and specific potential improvements of the game in this step. Step 3 (FGI): After a discussion with each paired partner, researchers conducted FGIs for 15-20 minutes on game experience and suggestions for game design improvement.
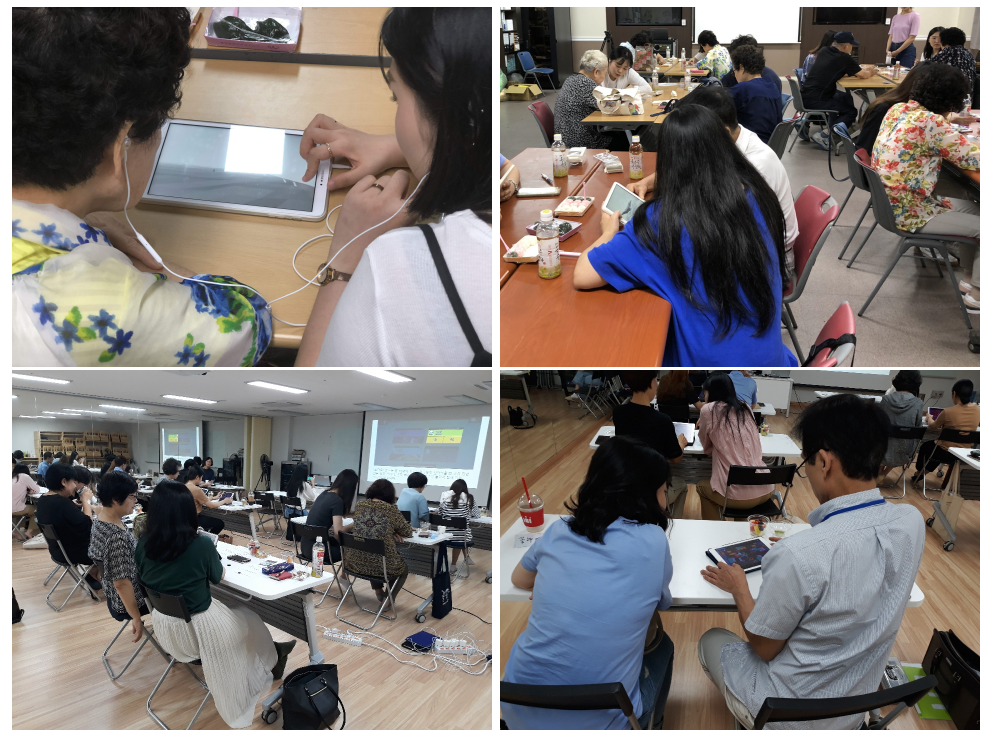

Fig. 2. Photographs from the "Games for Life Review" workshop (top row: senior welfare center; bottom row: education center for the $50+$ generation). 


\subsection{Data Analysis}

First, an inductive thematic analysis was conducted to identify themes in intergenerational communication (RQ1). we followed six phases of thematic analysis proposed by Braun and Clarke [6]. Transcript data were qualitatively analyzed using Nvivo, based on all recorded conversations during the activity between the senior participants and young partners. After reading the transcripts multiple times (step 1), the first author generated initial codes (step 2) and developed these codes into themes (step 3). Three authors reviewed and defined themes through discussion to reach an agreement (steps 4 and 5). These processes were continued throughout the analysis and writing processes (step 6).

Second, the analysis was expanded to include game elements that promote those themes. We analyzed the game considering the basic design elements of games, proposed by Macklin and Sharp [41]: goals, rules, play space, objects, actions, and players. We grouped basic elements, except players, into three categories: structural, metaphoric, and interactive. Then, we studied the link between the game's elements and communication themes (RQ2).

Third, We also identified the characteristics of senior participants who liked and disliked this game after examining each participant's satisfaction level and dialogues related to evaluations of the game. Moreover, We explored the difference in game experience between middle-aged adults and older adults. In addition, we analyzed the responses of young partners (RQ3). Lastly, we found implications for game design through participants' feedback on evaluations and suggestions (RQ4).

\section{FINDINGS}

\subsection{Results of Thematic Analysis}

Although there were many conversation topics, seven major themes were emerged most frequently. Table 2 shows definition and number of references and cases for each theme. Reference means the total number of times the theme is reported. Case means the number in which each theme emerged among 33 couples.

Table 2. Results of thematic analysis

\begin{tabular}{lllc}
\hline Theme & Definition & \multicolumn{2}{c}{ Reference (Case) } \\
SP & YP \\
\hline 1. Reminiscence & $\begin{array}{l}\text { Memories and feelings of events that occurred } \\
\text { in the one's past. }\end{array}$ & 208(32) & - \\
$\begin{array}{l}\text { 2. Future and death } \\
\text { preparation }\end{array}$ & $\begin{array}{l}\text { Preparation, determination, and thoughts of } \\
\text { one's future and death. }\end{array}$ & 28(19) & - \\
$\begin{array}{l}\text { 3. Life advice } \\
\text { Life advice derived from one's own experience } \\
\text { and wisdom. }\end{array}$ & $37(17)$ & - \\
$\begin{array}{l}\text { 4. Appreciation and } \\
\text { evaluation }\end{array}$ & $\begin{array}{l}\text { Appreciation and evaluation of the game and } \\
\text { activity including negative and positive responses. }\end{array}$ & $101(25)$ & $13(9)$ \\
$\begin{array}{l}\text { 5. Small talk involving } \\
\text { self-disclosure }\end{array}$ & $\begin{array}{l}\text { Small talk that reveals oneself, such as family } \\
\text { members, recent interests, etc. }\end{array}$ & $26(18)$ & $19(11)$ \\
$\begin{array}{l}\text { 6. Interpretation of } \\
\text { metaphors }\end{array}$ & $\begin{array}{l}\text { Interpretation of what an object or image in the } \\
\text { game means. }\end{array}$ & $106(29)$ & $173(33)$ \\
$\begin{array}{l}\text { 7. Explanation of } \\
\text { control methods }\end{array}$ & $\begin{array}{l}\text { Instructions on how to play the game by } \\
\text { controlling buttons. }\end{array}$ & - & $137(33)$ \\
\hline
\end{tabular}

$\mathrm{SP}=$ senior participant, $\mathrm{YP}=$ young partner. 
Themes that identified from only senior participants' discourse were "reminiscence, future and death preparation, and life advice." Themes that identified from only young partners were "explanation of control methods." In addition, "appreciation and evaluation, small talks involving self-disclosure, and interpretation of metaphors" were identified from the both groups.

\subsection{Game Elements Promoting Intergenerational Communication}

3.2.1 Structural Elements: Goals and Rules. The game "Long Journey of Life" consists of 10 stages that shows a person's life as a sailing boat. The goal of this game is moving a boat to the end and experiencing a life story from birth to death. There was a simple rule of going forward or backward, but players mostly move forward to see the next scenes. The title of the life period appeared at the beginning of each stage, for example, birth, childhood, family, and death. As participants played the Korean version, the title was in Korean text.

Related to the structural elements of the game, the "reminiscence" theme was frequently identified. Many senior participants' references of reminiscence were found in their responses to young partner's questions regarding the life period. The quotations below were originally spoken in Korean, but translated in English. SP stands for "senior participant," and YP stands for "young partner."

YP7 (female, 21): These are cranes. The boat has become a teenager. How was your teenage life? Were you still at war?

SP3 (male, 79): I was a teenager at the beginning of the Korean War. We left home and went to the shelter. Even after the ceasefire, there was no desk at school. While studying, we just hid when we heard airplanes. (Reference during co-playing)

There were certain later stages, such as children's independence and late age, that some of the senior participants had not actually experienced in their real lives. In these stages, participants told stories based on imagination and talked about their future. We identified these references as "future and death preparation" theme.

YP8 (female, 21): How did you feel in the stage of late age?

SP24 (male, 55): When old age comes, I guess every moment will be difficult. I want to empty my mind and think positively. (Reference during paired discussion)

In the last stage of the game, the player boat said goodbye to the family, fell off the cliff, and died. Two senior participants shed tears during this scene. The game ends as the boat sails through its past and reaches a location where many other boats are seen. Death is generally a serious and difficult topic of conversation. However, participants were able to talk freely about how to die well and how to prepare for it. We also identified these references as the "future and death preparation" theme.

SP22 (female, 57): I thought about how I could live the rest of my life and how I can age well. I was thinking only about me. However, (the last scene) a sudden farewell? That made me cry, because I had not thought about it yet. I do not plan to suddenly say goodbye to the people around me. It should not be just for me. I have to prepare for death, say a good farewell to others-that's the plan I should make. (Reference during FGI)

SP3 (male, 79): I will not have prolonged treatment. I do not want to make it difficult for my children. I have to sign in advance. (Reference during paired discussion)

During and after playing the game, participants expressed that the game seemed to show their lives. This dialogue was identified as the "appreciation and evaluation" theme.

SP15 (female, 78): This was similar to the flow of life. From birth, raising a child, and sending him to get married. Becoming old... That was life itself. (Reference during FGI) 
However, some participants complained that the game had only one linear story and suggested to provide options to choose alternative stories. The game introduces the story of a married person with one child; in reality, life experiences have far more variation.

SP30 (female, 51): I have twins, but there was only one child in the game. I wish I had more options to customize. (Reference during FGI)

SP20 (male, 64): As the game was very simple, I would not play it many times. If the game offers more choices, I would like to choose a path that is different from my real life and play it again. (Reference during FGI)

3.2.2 Metaphoric Elements: Play Space and Objects. This game depicted a person's life as a boat sailing in the ocean, and each level had a different play space atmosphere that included metaphorized objects for the player to enjoy interpreting. The theme "interpretation of metaphors" was frequently emerged related to play space and objects. Young partners mainly took a role of explaining the meanings of the metaphors, but senior participants also interpreted their own meanings. Senior participants empathized with the interpretations and applied the metaphors to their lives, leading to "reminiscence." In particular, many participants empathized with the scene that represents life hardships as blocked container boxes, as well as scenes that depict life events as changing weather.

YP9 (female, 23): Now, it's adulthood.

SP6 (female, 69): There are rainy days, cloudy days, and sunny days. This game represents our life. Frankly, there were many sunny days, but people think only about their hardships. After all, I think there were more good days, and I am comfortable and happy now because I lived so hard. I think it's fruitful that I worked hard. I am satisfied now. (Reference during co-playing)

Some objects evoked past memories for senior participants accurately or provided clues to young partners for asking proper questions. For example, after the player boat passed the adulthood stage, it met a lover boat. Here, in nine instances, young partners were asked about how they met their spouses and how they felt at that time.

YP9 (female, 23): This boat is a wife.

SP28 (male, 63): This reminded me of when we got married.

YP9: How did you feel at that time?

SP28: Everyone is happy for the first time. We went on a honeymoon and I was happy...

was happy...

YP9: Why is it past tense? (laugh) (Reference during co-playing)

Furthermore, in three cases of this scene, senior participants shared life advice with young partners on how to choose good spouses based on their past experience. One participant even spoke out against living together before marriage. This led to discussions with young partners. We identified these references as the "life advice" theme.

YP6 (female, 21): What was your first impression of your husband?

SP8 (female, 72): The first impression is not important, but personality is more important.

I liked neat and handsome guys when I was young...

YP6: Same with me!

SP8: But appearance was not important to live together. He just cared about his clothes and was not faithful to the family. (Reference during co-playing)

In addition, we found "reminiscence" themes from seven senior participants in the scene that the boat rode the merry-go-round with the child boat. They recalled memories of having fun with their kids during playing. 
YP7 (female, 21): Did you go to an amusement park many times when your child was young?

SP29 (female, 59): I did not like riding a roller coaster, but my child loved it. I lived there after buying an annual pass. (Reference during co-playing)

Some objects were not successful in creating empathy and invoking recollection. For example, many senior participants could not empathize with the scene where the boat passed a teddy bear and wooden horse in the childhood stage because they did not have fancy toys in their childhood.

YP3 (female, 23): Did you play with these kinds of dolls when you were young?

SP27 (female, 65): We did not have toys like this. These things are what I see now while raising my granddaughter. (Reference during co-playing)

SP14 (female, 79): We could not even imagine having a toy; we just played with five stones-a Korean traditional game using little rocks-in the yard. (Reference during coplaying)

In addition, some objects had ambiguous meanings and could be interpreted in many ways. For example, the late age scene, in which the world turns upside down and the player boat sails in the opposite direction, was interpreted by some couples as looking back to the past, while some interpreted it as the physical, mental, and relational loss of old age. Moreover, senior participants who interpreted this scene as a decline expressed fear about illness in old age.

SP21 (female, 57): Is this game telling us to live a life of reflection? Looking back... Preparing for the end of life? (Reference during co-playing)

SP32 (female, 52): Does this mean a decline in physical functioning? We should feel at ease when we are old; how can it be shaken like this? (Reference during co-playing)

SP1 (male, 73): When the boat flipped upside down, it was heartbreaking. If I get sick like that someday, how much it will hurt... when we turn over... (Reference during FGI)

As a theme of "appreciation and evaluation," two senior participants evaluated the game's portrayal of old age as abbreviated, simple, and depressing. They suggested that a thorough understanding of older people's lives is necessary to provide ideal games for life review, recognizing and incorporating factors to help players convey more positive emotions, such as comfort, joy, and action. In addition, one senior participant pointed out that images in this game are based on Western culture, and suggested a design that would be more sympathetic to the player's cultural context.

SP32 (female, 52): I do not know how old the people who made this game are, but I think they lack an understanding of old age. Compared to the diversity of the first half, I do not think the second half of life is as simple as portrayed in this game. (Reference during FGI) SP29 (female, 59): The image of old age was expressed as confused and negative in this game. When expressing old age, I hope it will be more adaptable and natural. It would be great if the game is based on the emotions and characteristics of our own culture, pop music, history. (Reference during FGI)

In the stage of adolescence and adulthood, two senior participants asked about the young partner's current situation. When the subtitle of "vacation" appeared, one couple talked about their vacation plans for the year. Moreover, they exchanged some personal information, such as numbers of family members, where they lived, recent interests, etc. We identified these conversations as "small talk involving self-disclosure."

In addition, the game featured natural sounds of waves, rain, radio, and peaceful melodies. Five participants gave a positive evaluation about the sound. However, two participants evaluated that the music is too simple. 
SP32 (female, 52): This feels great with the sound of water. ASMR.

YP4 (male, 23): You might fall asleep doing it.

SP32: It's like I'm doing meditation.

YP4 (male, 23): If you turn off the lights...

SP32: I would fall asleep soon. (laugh) I think I'll have a good dream. (Reference during co-playing)

3.2.3 Interactive Elements: Actions. Although the game has simple controls, the player performs a continuous action to move to the next scene. It was different from watching a short animation passively, as players themselves must press the button. For example, senior participants had to repeatedly move back and forth to bump into a lover boat several times to produce a baby boat and adjust the speed to match the child boat's pace. Senior participants preferred to understand how to play before beginning, rather than learning while playing. Senior participants often asked for guidance and noted that they would not have related to the game or been able to figure out how to play it alone. It was also challenging for senior participants to reminiscence without guidance from young partners, who explained the controls of the game and the meaning of the objects. Many communication patterns were identified, as senior participants asked how to control and young partners explained. We called this theme as "explanation of control methods."

For example, regarding the action that the player boat and the lover boat bump into each other and made sparks before marriage, the communication theme started with "explanations of the control methods" by the young partners, followed by "interpretation of metaphors" and "reminiscence" by the senior participants. According to the interpretation of this action, different subjects of reminiscence were followed. One couple interpreted the scene as a conflict between lovers, and the senior participant recollected the memories of her sister's marriage story.

YP8 (female, 21): Go back and make a spark!

SP4 (female, 67): There are many conflicts before marriage.

YP8: I think this shows the feeling of frustration before marriage. Men and women have a relationship and break up repeatedly before marriage.

SP4: It happened to my sister. It was serious. She wanted to marry someone who had the same surname as her, which was conventionally unacceptable at that time. Although my father disagreed with the marriage, they are living well, with a child who has now attends middle school. (Reference during co-playing)

Another couple interpreted this scene as a flame of love, and the senior participant was reminded of her marriage.

YP2 (male, 23): You need to bump into each other several times. Go backward and...

SP22 (female, 57): Oh, I see. Need a deep connection?

YP2: Yes.

SP22: It's marvelous. When we fall in love, sometimes we feel a deep connection, and sometimes we do not. I dated for six years before I got married. (Reference during coplaying)

In addition, one couple lost the child boat because the senior participant moved too fast. $\mathrm{He}$ recalled the memory that he had lost his child by connecting the play situation to the real event.

YP9 (female, 23): Oh, here it is. You have to take him. Now make sure he is following you when you go forward.

SP28 (male, 63): I have lost my child and found it too.

YP9: Really?

SP28: Yes. You know what? Kids always move forward when they get lost. 
YP9: Yes, I know.

SP28: My wife tells me that story from time to time. My wife went straight for a long time and found him. (Reference during co-playing)

One senior participant offered a young partner "life advice" about staying a little apart to improve the relationship when she controlled the boat.

SP32 (female, 52): This degree of distance is good. It is not good to stick together between lovers. (Reference during co-playing)

As for "appreciation and evaluation of game design", one senior participant said it was good because the control was easy and not burdensome. However, one senior participant said that this game was not satisfactory because it lacked active actions.

\subsection{Differences in Responses by Participants}

3.3.1 Differences Between the Highly Satisfied and Less Satisfied Groups. After the session, senior participants' satisfaction was surveyed by the question, "How satisfied are you with this game?" Answers were measured on a 5 -point scale ( 1 = very unsatisfied; $2=$ unsatisfied; 3 = average; $4=$ satisfied; 5 = very satisfied). The mean satisfaction level of the game was 3.33 , and the standard deviation was 1.29. It is assumed that the standard deviation was large due to the significant variation in participants' satisfaction with the game. Senior participants who enjoyed the interpretation process and sympathized with the metaphors in the game expressed high satisfaction.

SP22 (female, 57): This game gives me a chance to see myself in an objective way. It was good feeling to see my life newly and objectively. (Reference during FGI)

SP30 (female, 59): It was nice experience sharing emotions with a partner who I did not know well before. Despite the short time period, we enjoyed open communication from the beginning. I felt comfortable telling my stories to the partners as I took personal questions indirectly by reflecting on the scenes in the game. (Reference during FGI)

SP8 (female, 72): When I was younger, I cried a lot because I had only daughters. They are now independent, and my life is happy. I feel a sense of achievement about being able to tell my story. I agree that life is like a sailing boat. (Reference during FGI)

On the other hand, senior participants who could not sympathize with metaphors and those who preferred realistic designs and high quality of active actions were less satisfied. In addition, participants who did not like to share their personal stories with others or a participant who suffered from recollection of the past were also less satisfied. The following quotes were recorded from participants who rated "very unsatisfied."

SP20 (male, 64): It was not interesting to me. I could not understand the meaning of the images without the young partner's explanation. I do not understand what the game developer wanted to say. The technology and graphics were too simple, as if it was made in the 60s. (Reference during FGI)

SP23 (male, 59): As I grew older, I became increasingly emotionally dry. These days, I cannot really feel anything, despite how touching the video was. I felt like a participant in a psychological test rather than a game player. (Reference during FGI)

SP12 (female, 74): Things were good only before marriage... I attended hairdressing school at that time. That was the only good time... I hope this boat will just sink, plop.

YP2 (male, 23): Why?

SP12: I don't know. Why am I sad to see this?... If this is my ship, I'd just like it to sink here. Married now? Oh, this isn't good. I don't want to remember. (Reference during co-playing) 
3.3.2 Differences Between the Middle-aged and Older Adult Groups. We could not find significant difference in satisfaction level among middle-aged adults (ages 50-65) and older adults (ages 66-85), but we found some differences in the response to gameplay between two groups. First, middle-aged and older adults showed differences in the content of reminiscences in their youth. This is due the wide in historical and cultural environments of both generations. In the 50s and 60s, when the older adults were young, Korea went through a war, and everyone suffered from a lack of resources. On the other hand, the 70 s and 80 s, when the middle-aged adults spent their youth, were a period of rapid economic growth in a developing country.

In the reminiscence of older adults, there were many stories about how they had a hard time and how they overcame it. There were also many expressions of regret for not receiving sufficient education.

SP18 (female, 75): We do not have any joyful memories with our children. My husband and I were too busy because we had no money. However, I am satisfied now because my three sons grew well without any troublemaking. (Reference during co-playing)

SP17 (female, 80): Thorny path... gravel road... I passed them all. Raising five children cannot be easy! I am happy now. (Reference during co-playing)

SP19 (female, 76): I would like to learn something new related to music. It is so thrilling! I did not attend a lot of school, so I tried my best to learn while sitting in the front. (Reference during paired discussion)

The young partners were interested in romantic relationships and asked many questions about them. However, as per tradition, most of the older adults had experienced arranged marriages by matchmaking, and there were few romantic stories. In the reminiscence of middle-aged adults, participants recalled pleasant memories of romantic relationships, but added that the marriage was not a fantasy, but in fact, reality.

SP22 (female, 57): Now married! I guess only three days were sweet. There are too many obstacles around us to just sweet each other. This makes me think a lot... (Reference during co-playing)

Most of the older adults were now in the old age stage after their children's independence, and most middle-aged participants were still in the stage prior to their children being independent Thus, different responses emerged from the gameplay at this stage. While the older adults talked extensively about sadness and relieved feelings, the middle-aged adults expressed their desire for their children's independence and envy of the game character.

YP7 (female, 21): What did you feel when your children were independent?

SP3 (male, 79): I cried a lot at my youngest daughter's wedding. My heart was crying, strangely... (Reference during paired discussion)

YP4 (male, 23): This scene shows the child's independence after marrying.

SP32 (female, 52): I will be relieved. It will be happy! He is not even alone and now has a partner. I envy them.

YP4: Do you want your child to become independent soon?

SP32: Of course! (Reference during co-playing)

Table 3 shows the frequency of each theme in two groups. The "reminiscence" theme tended to appear more often in the older adult group than it did in the middle-aged group. These older adult participants were inclined to share personal history and traditional values with young partners based on their intimacy. Older adults acquire a sense of generativity during middle age and are eager to contribute to society after the stagnation stage, during which they focus mostly on themselves or family $[24,29]$. On the other hand, middle-aged adults reported more "appreciation and evaluation." 
Table 3. Descriptive statistics for the frequency of each theme in two groups

\begin{tabular}{ccccccc}
\hline \multirow{2}{*}{ Communication Theme } & \multicolumn{2}{c}{ Middle-aged adults (N=14) } & & \multicolumn{2}{c}{ Older adults (N=19) } \\
\cline { 2 - 3 } \cline { 6 - 7 } & Reference & Mean (SD) & & Reference & Mean (SD) \\
\hline Reminiscence & 71 & $5.07(3.08)$ & & 137 & $7.21(4.69)$ \\
Future and death preparation & 14 & $1.00(0.93)$ & & 14 & $0.74(0.85)$ \\
Life advice & 10 & $0.77(1.85)$ & & 27 & $1.50(1.86)$ \\
Appreciation and evaluation & 62 & $4.43(3.81)$ & & 39 & $2.05(1.67)$ \\
Small talks involving self-disclosure & 16 & $1.14(1.46)$ & & 10 & $0.53(0.60)$ \\
Interpretation of metaphors & 56 & $4.00(3.27)$ & & 50 & $2.63(2.58)$ \\
\hline
\end{tabular}

Since the middle-aged group had higher levels of digital literacy, their opinions were more specific. Also, they suggested more options to improve the game design.

3.3.3 Response of Young Adult Partners. In the workshop, young partners used various communication strategies to elicit successful reminiscence from senior participants: asking appropriate questions in connection with game scenes, listening carefully, asking additional questions that led to more specific memories, empathizing with older people's feelings and comforting them, and telling their own stories. However, we also found personal differences in the capabilities of young partners communicating with seniors. There were young partners who asked questions passively or unnaturally.

Young partners were interested in senior participants' stories about phenomena the young partners had never experienced, such as wars and past customs, and asked to hear more stories. Most young partners presented positive feedback on the activity during the debriefing session, which only young partners participated in.

YP4 (male, 23): It was the most interesting game I played during the IPW.

YP6 (female, 21): I kept thinking about my partner's story. I feel like I will be like her in the future. I gained a better understanding of her life through this experience.

However, it was an awkward experience for young partners who did not have a chance to establish a close rapport with a partner before the activity. Most couples had an intimate relationship already, but there were two cases of couples who barely knew each other beforehand. In addition, one young partner said it was an embarrassing experience for her to interact with the senior participant, who seemed reluctant to talk about personal stories.

YP7 (female, 21): The first session was okay. However, in the second session, I played with senior participant who had never been a couple before. It was a bit difficult to ask questions. YP1 (female, 19): He didn't even want to talk about normal questions such as, "Are you married?", "Do you have a child?”... He said it is like taking a psychological test, so I felt embarrassed.

\section{DISCUSSION}

\subsection{Using Games for Promoting Intergenerational Communication}

4.1.1 Creating a Comfortable and Enjoyable Atmosphere. The findings of this study indicate that games provided participants with opportunities to communicate and practice life review comfortably. The life review process can sometimes be unpleasant, depending on the personality of the reviewer, as it involves revealing personal stories to others; reviewers can become defensive or take offense 
to the listener's questions. However, in the gameplay workshop, participants responded positively to the process because they were able to enjoy the experience through a game involving text, video, and music, rather than a one-on-one interview in a closed room. This was possible because people usually perceived playing games as fun and enjoyable activities. Intergenerational play facilitates interactions between two generations and develops positive intergenerational perceptions $[16,59,60]$. Young partners could generate proper questions using metaphors in the game, such as "Do you remember anything associated with this scene?" and "When your boat said goodbye and passed away, what were your thoughts?" Through the questions related to the game, participants had opportunities to review their lives objectively from a distance. Moreover, conversation topics were naturally refreshed at each stage, as gameplay was prioritized over storytelling.

However, there were also some senior participants who were dissatisfied with the activity because they did not enjoy the self-disclosure. Hence, this activity will be appropriate when participants have high levels of openness and when couples have high levels of intimacy. In addition, ones who refuse to recall past events or have experienced trauma may be better helped by professional treatment than the intergenerational life review activities.

4.1.2 Promoting Symmetrical Interaction in Intergenerational Relationships. We found the possibility of having a balance of conversation wherein young people can teach seniors game literacy, and seniors can provide life advice from personal experience. The study of intergenerational learning (IGL) also stressed that social interactions should not be a one-way process, but should benefit both generations [48]. Previous studies on intergenerational playing highlighted an imbalance in interaction $[2,57,59,60]$. This is because young people are more proficient in using digital games and seniors usually ask questions; young people always play a role in teaching the senior participants how to use the games. As in previous studies [60], undergraduate students in the workshop mainly served to provide explanations and guide older adults. However, in the gamebased life review activity, a more reciprocal relationship was possible because the senior participants were able to actively convey cultural values and wisdom from their life experiences.

In addition, using digital games for life review activities can add game-related conversations to stories about reminiscence. Zucchero [61] conducted a structured life review as an intergenerational activity based on a community program. It was similar to a traditional life review activity and conducted through one-on-one interviews. The study found similar content themes with our results, such as reminiscence, and students listened to older adults' stories as older adults served as a role model. However, life review activities using digital games have found new themes related to games, such as metaphor interpretation, explanation of control methods, and appreciation and evaluation

4.1.3 Role of Young Adult Partners. It was also found that the role of young partners is critical in life review activity in this study. They asked questions to elicit reminiscence, explained metaphors in the game, and guided game playing. Despite relatively simple gaming controls, senior participants mentioned that they would have experienced difficulties enjoying the game without the help of young partners. Emotional interaction between a reviewer and a listener plays an important role in reminiscence [34]. In the workshop, young partners used various communication strategies to elicit successful reminiscence of senior participants, but there were personal differences in capabilities as facilitators. Therefore, successful life review activities will require pre-sessions for young partners to train game play methods and effective communication strategies.

\subsection{Implications for Game Design}

4.2.1 Connecting Real Life to Encourage Self-disclosure and Meaningful Conversation. We found that reminiscence was promoted well when participants understood and sympathized the meaning of metaphors in the game. As the life metaphoric game "Long Journey of Life" provided metaphoric 
elements connected to real life, it could encourage conversations related to personal stories. Likewise, Siyahhan and Gee [50] investigated conversation and behavior during intergenerational gameplaying in the family and found that there were certain games that made real-life connections easier and promoted more self-disclosure communication. For example, when parents and children played "The Sims 3," they could share personality traits, hobbies, and aspirations in the process of creating and decorating objects in the game. Parents used their real-life experiences to make better decisions in the game. Intergenerational playing can promote self-disclosure and relationship closeness [44]. People usually talk about the game itself during intergenerational play; subjects can naturally change to small talk and the discussion of important life issues [43]. It is assumed that game designs linked to real life will help promote intergenerational life review. According to Räisänen and colleagues [46], successful game design for IGL also emphasized that the game could mimic situations in the real world and make players think more about their actions. Moreover, it was recommended that fictional stories have no clear right or wrong answers.

4.2.2 Application of Interactive Storytelling. Erikson's stage theory has been criticized for generalizing various forms of aging into a single process [29]. Participants in the present study similarly criticized the "Long Journey of Life" for providing experiences from only one story. Games intended to support life review should reflect a variety of real-life experiences rather than stereotypical lifestyles. If life review games apply an interactive storytelling system, players could choose the game stories that best fit their own life experiences. Interactive storytelling techniques can encourage players to play again and think about the paths they did not take by making other choices. This requires a more complex and sophisticated story design. Further research is needed to examine the psychological impact of indirectly experiencing alternative life paths through gameplay.

4.2.3 Expressing the Life of Old Age in a Richer and More Active Manner. Older people's lives should be portrayed more actively in life review games. Some participants were dissatisfied with the scenes of old age because they were expressed as abbreviated, simple, and depressing. "Gerontoludic manifesto" by De Schutter [21] emphasized heterogeneity over stereotyping with criticism that many commercial games and academic literature focused on ageing as a process of decline and debilitation. We also suggest that the ordinary life in old age should not be described in excessively static, passive, negative, or gloomy terms. Story designers must have a deep understanding of older life to create rich narratives. Careful consideration of minority groups is also necessary; players were able to empathize better with metaphors similar to their own cultural backgrounds. Lindley, Harper, and Sellen [39] argued that new technologies are often developed based on simple assumptions regarding older adults. They presented more complex reality in later life in line with the perspectives of older adults. Game design workshops that allow older adults to participate in the design process could be helpful in identifying the ideal approach and reflecting immediate feedback $[4,47]$.

4.2.4 Promoting Integrative Resilience and Personal Growth. Life review games for elderly players should be designed to include various life stages and provide a chance to reflect a player's life in integrative ways. To do this, they must convey rich stories recalling moments of challenges, hardships, and happiness while balancing with each stage. One participant mentioned that through the game play, she could recollect everything that she had been through. Another participant said that she recalled hardships in the past to raise five children, and was proud herself and decided to enjoy the rest of her life at the end. Games provide older adults with pleasurable emotional outcomes, personal growth, and perceived benefits [22]. From a lifespan perspective, meaning in digital games is derived from players' desire to facilitate connectedness, cultivate themselves and 
others, and contribute to society [23]. Although life reivew games do not focus primarily on fun, successful life review games support personal growth and foster healthy relationships.

\section{CONCLUSION}

This study revealed that life metaphoric games could be useful tools to promote various communication themes during intergenerational life review activities. However, some limitations of this study should be considered. First, although a dyadic approach was adopted, but there was the imbalance in numbers between senior participants and young partners. Moreover, the responses of young partners were not collected and analyzed sufficiently in comparison to the senior participants. Second, we could not include participants in familial relationships, even though such activities are well-suited for people in familial relationships. Third, because the game was not targeted at older adults or intentionally designed for life review, accessibility with respect to the size of text and objects was unsatisfactory for some participants. Finally, the traditional structured life review is performed via an in-depth interview about each life stage through eight meetings [31]. However, this workshop was conducted as a one-day session, and the gameplay times were short. As the workshop was conducted over a short period, changes in the relationship between the reviewer and listener could not be carefully observed. Despite the limitations, the study attempted a new activity by combining intergenerational play and life review. Future research is needed to investigate whether these activities can contribute to promoting successful aging and intergenerational relationships. In addition, it is necessary to expand the scope of the study to include familial relationships.

Although games are primarily designed for young adults, many middle- and older-aged players have continuously played them since their childhood. The demand for games among such players has resulted in a mature gaming industry. Successful life review games deliver players with rich impressions through reminiscence associated with the game's stories. In addition, they can help older adults take on a storytelling role, allowing them to continually contribute to the community and maintain their social status. These findings can support future researchers and designers of games for life review.

\section{ACKNOWLEDGMENTS}

The authors would like to thank Li Yang (PINX Studio), the developer of the game "A Long Journey of Life." The authors also appreciate senior citizens and young adult partners who participated in the Intergenerational Play Workshop held by KAIST in 2019. This research was supported by the Ministry of Culture, Sports and Tourism (MCST) and Korea Creative Content Agency (KOCCA) through the Culture Technology (CT) Research and Development Program (Project No: R2019020010).

\section{REFERENCES}

[1] [n.d.]. Anecdote. Retrieved June 1, 2020 from https://www.cdsboutique.com/en/anecdote-the-reminiscing-game-forseniors.html

[2] Vero Vanden Abeele and Bob De Schutter. 2010. Designing intergenerational play via enactive interaction, competition and acceleration. Personal and Ubiquitous Computing 14, 5 (2010), 425-433. https://doi.org/10.1007/s00779-009-0262-3

[3] Jason C Allaire, Anne Collins McLaughlin, Amanda Trujillo, Laura A Whitlock, Landon LaPorte, and Maribeth Gandy. 2013. Successful aging through digital games: Socioemotional differences between older adult gamers and non-gamers. Computers in Human Behavior 29, 4 (2013), 1302-1306. https://doi.org/10.1016/j.chb.2013.01.014

[4] Steven Baker, Jenny Waycott, Romina Carrasco, Thuong Hoang, and Frank Vetere. 2019. Exploring the Design of Social VR Experiences with Older Adults. In Proceedings of the 2019 on Designing Interactive Systems Conference. ACM, NY, USA, 303-315. https://doi.org/10.1145/3322276.3322361

[5] Samuel Benveniste, Pierre Jouvelot, Benoît Pin, and Renaud Péquignot. 2012. The MINWii project: renarcissization of patients suffering from Alzheimer's disease through video game-based music therapy. Entertainment Computing 3, 4 (2012), 111-120. https://doi.org/10.1016/j.entcom.2011.12.004 
[6] Virginia Braun and Victoria Clarke. 2006. Using thematic analysis in psychology. Qualitative research in psychology 3, 2 (2006), 77-101.

[7] Robin N Brewer and Jasmine Jones. 2015. Pinteresce: exploring reminiscence as an incentive to digital reciprocity for older adults. In Proceedings of the 18th ACM conference companion on computer supported cooperative work \& social computing. ACM, NY, USA, 243-246. https://doi.org/10.1145/2685553.2699017

[8] Robert N Butler. 1963. The life review: An interpretation of reminiscence in the aged. Psychiatry 26, 1 (1963), 65-76.

[9] Robert N Butler. 1974. Successful aging and the role of the life review. Fournal of the American geriatrics Society 22, 12 (1974), 529-535. https://doi.org/10.1111/j.1532-5415.1974.tb04823.x

[10] Alessia Cadamuro and Valentijn Visch. 2013. 'What Remains?': A Persuasive Story Telling Game. In Games for Health. Springer, Wiesbaden, GER, 153-160. https://doi.org/10.1007/978-3-658-02897-8_11

[11] Niamh Caprani, Nuala Dwyer, Kim Harrison, and Karen O’Brien. 2005. Remember when: development of an interactive reminiscence device. In CHI'05 Extended Abstracts on Human Factors in Computing Systems. ACM, NY, USA, 2070-2073. https://doi.org/10.1145/1056808.1057101

[12] Kaige Chang, Ning An, Jian Qi, Rongean Li, Sue Levkoff, Hongtu Chen, and Peng Li. 2013. Food stamps: a reminiscence therapy tablet game for Chinese seniors. In 2013 ICME International Conference on Complex Medical Engineering. IEEE, 556-561. https://doi.org/10.1109/ICCME.2013.6548312

[13] Ya-Fang Cheng, Szu-Yang Cho, and Hsien-Hui Tang. 2017. Memoir Monopoly: Improving Rehabilitation Activities for Elderly People with Dementia. User Experience Magazine 17, 1 (2017). Retrieved January 10, 2020 from http: //uxpamagazine.org/memoir-monopoly/

[14] Kai-Jo Chiang, Hsin Chu, Hsiu-Ju Chang, Min-Huey Chung, Chung-Hua Chen, Hung-Yi Chiou, and Kuei-Ru Chou. 2010. The effects of reminiscence therapy on psychological well-being, depression, and loneliness among the institutionalized aged. International fournal of Geriatric Psychiatry: A journal of the psychiatry of late life and allied sciences 25, 4 (2010), 380-388. https://doi.org/10.1002/gps.2350

[15] Tracy Chippendale and Marie Boltz. 2015. Living Legends: students' responses to an intergenerational life review writing program. Journal of the American Geriatrics Society 63, 4 (2015), 782-788. https://doi.org/10.1111/jgs.13236

[16] Puay-Hoe Chua, Younbo Jung, May O Lwin, and Yin-Leng Theng. 2013. Let's play together: Effects of video-game play on intergenerational perceptions among youth and elderly participants. Computers in Human Behavior 29, 6 (2013), 2303-2311. https://doi.org/10.1016/j.chb.2013.04.037

[17] Thecla Damianakis, Masashi Crete-Nishihata, Karen L Smith, Ronald M Baecker, and Elsa Marziali. 2009. The psychosocial impacts of multimedia biographies on persons with cognitive impairments. The Gerontologist 50, 1 (2009), 23-35. https://doi.org/10.1093/geront/gnp104

[18] Teresa De la Hera, Eugène Loos, Monique Simons, and Joleen Blom. 2017. Benefits and factors influencing the design of intergenerational digital games: a systematic literature review. Societies 7, 3 (2017), 18. https://doi.org/10.3390/ soc7030018

[19] Jos De Mul. 2015. The game of life: Narrative and ludic identity formation in computer games. In Representations of Internarrative Identity. Palgrave Macmillan, London, UK, 159-187. https://doi.org/10.1057/9781137462534_10

[20] Bob De Schutter. 2011. Never too old to play: The appeal of digital games to an older audience. Games and Culture 6, 2 (2011), 155-170. https://doi.org/10.1177/1555412010364978

[21] Bob De Schutter and Vero Vanden Abeele. 2015. Towards a gerontoludic manifesto. Anthropology \& Aging 36, 2 (2015), 112-120. https://doi.org/10.5195/aa.2015.104

[22] Bob De Schutter and Julie A Brown. 2016. Digital games as a source of enjoyment in later life. Games and Culture 11, 1-2 (2016), 28-52. https://doi.org/10.1177/1555412015594273

[23] Bob De Schutter and Vero Vanden Abeele. 2010. Designing meaningful play within the psycho-social context of older adults. In Proceedings of the 3rd International Conference on Fun and Games. ACM, NY, USA, 84-93. https: //doi.org/10.1145/1823818.1823827

[24] Erik H Erikson and Joan M Erikson. 1998. The life cycle completed (extended version). WW Norton \& Company, NY, USA.

[25] Brian J Gajadhar, Yvonne AW De Kort, and Wijnand A Ijsselsteijn. 2008. Shared fun is doubled fun: player enjoyment as a function of social setting. In International Conference on Fun and Games. Springer, Berlin, Heidelberg, GER, 106-117. https://doi.org/10.1007/978-3-540-88322-7_11

[26] Alice Good, Clare Wilson, Claire Ancient, and Arunasalam Sambhanthan. 2013. A proposal to support wellbeing in people with borderline personality disorder: applying reminiscent theory in a mobile app. arXiv preprint arXiv:1302.5200 (2013).

[27] Thaddeus Griebel. 2006. Self-portrayal in a simulated life: Projecting personality and values in The Sims 2. Game Studies 6, 1 (2006).

[28] Bin Guo, Zhiwen Yu, Xingshe Zhou, and Daqing Zhang. 2013. MemPhone: From personal memory aid to community memory sharing using mobile tagging. In 2013 IEEE International Conference on Pervasive Computing and 
Communications Workshops (PERCOM Workshops). IEEE, 332-335. https://doi.org/10.1109/PerComW.2013.6529509

[29] David Haber. 2006. Life review: Implementation, theory, research, and therapy. The International fournal of Aging and Human Development 63, 2 (2006), 153-171. https://doi.org/10.2190/DA9G-RHK5-N9JP-T6CC

[30] Barbara K Haight. 1988. The therapeutic role of a structured life review process in homebound elderly subjects. Journal of gerontology 43, 2 (1988), P40-P44. https://doi.org/10.1093/geronj/43.2.P40

[31] Barbara K Haight and Barrett S Haight. 2007. The handbook of structured life review. Health Professions Press, Baltimore, MD, USA.

[32] Amanda K Hall, Enmanuel Chavarria, Vasana Maneeratana, Beth H Chaney, and Jay M Bernhardt. 2012. Health benefits of digital videogames for older adults: a systematic review of the literature. Games for Health: Research, Development, and Clinical Applications 1, 6 (2012), 402-410. https://doi.org/10.1089/g4h.2012.0046

[33] Aimee V Hamel, Tai L Sims, Dan Klassen, Thomas Havey, and Joseph E Gaugler. 2016. Memory matters: A mixedmethods feasibility study of a mobile aid to stimulate reminiscence in individuals with memory loss. Fournal of gerontological nursing 42, 7 (2016), 15-24. https://doi.org/10.3928/00989134-20160201-04

[34] Berit Ingersoll-Dayton, Nancy Kropf, Ruth Campbell, and Michael Parker. 2019. A systematic review of dyadic approaches to reminiscence and life review among older adults. Aging \& mental health 23, 9 (2019), 1074-1085 https://doi.org/10.1080/13607863.2018.1555696

[35] Vaiva Kalnikaite and Steve Whittaker. 2011. A saunter down memory lane: Digital reflection on personal mementos. International fournal of Human-Computer Studies 69, 5 (2011), 298-310. https://doi.org/10.1016/j.ijhcs.2010.12.004

[36] Linda K Kaye and Jo Bryce. 2012. Putting the fun factor into gaming: The influence of social contexts on the experiences of playing videogames. International fournal of Internet Science 7, 1 (2012), 24-38.

[37] Amanda Lazar, Hilaire Thompson, and George Demiris. 2014. A systematic review of the use of technology for reminiscence therapy. Health education \& behavior 41, 1_suppl (2014), 51S-61S. https://doi.org/10.1177/1090198114537067

[38] Hung-Chi Lee, Ya Fang Cheng, Szu Yang Cho, Hsien-Hui Tang, Jane Hsu, and Chien-Hsiung Chen. 2014. Picgo: designing reminiscence and storytelling for the elderly with photo annotation. In Proceedings of the 2014 companion publication on Designing interactive systems. ACM, NY, USA, 9-12. https://doi.org/10.1145/2598784.2602769

[39] Siân E Lindley, Richard Harper, and Abigail Sellen. 2008. Designing for elders: exploring the complexity of relationships in later life. People and Computers XXII Culture, Creativity, Interaction 22 (2008), 77-86. https://doi.org/10.14236/ewic/ HCI2008.8

[40] Naemi Luckner, Fares Kayali, Oliver Hödl, Peter Purgathofer, Geraldine Fitzpatrick, Erika Mosor, Daniela SchlagerJaschky, and Tanja Stamm. 2013. From research to design-sketching a game to trigger reminiscence in older adults. In International Conference on Human Factors in Computing and Informatics. Springer, Berlin, Heidelberg, GER, 617-624. https://doi.org/10.1007/978-3-642-39062-3_41

[41] Colleen Macklin and John Sharp. 2016. Games, Design and Play: A detailed approach to iterative game design. AddisonWesley Professional, Boston, MA, USA.

[42] Assunta Matassa and Amon Rapp. 2015. Map: making: designing a mobile application for enhancing memories' retrieval. In Proceedings of the 17th International Conference on Human-Computer Interaction with Mobile Devices and Services Adjunct. ACM, NY, USA, 994-1001. https://doi.org/10.1145/2786567.2794318

[43] Sanela Osmanovic and Loretta Pecchioni. 2016. Beyond entertainment: motivations and outcomes of video game playing by older adults and their younger family members. Games and Culture 11, 1-2 (2016), 130-149. https: //doi.org/10.1177/1555412015602819

[44] Sanela Osmanovic and Loretta L Pecchioni. 2019. Playing with Words: The Experience of Self-disclosure in Intergenerational Gaming. In International Conference on Human-Computer Interaction. Springer, Cham, 189-203. https://doi.org/10.1007/978-3-030-22015-0_15

[45] Celia Pearce. 2008. The truth about baby boomer gamers: A study of over-forty computer game players. Games and Culture 3, 2 (2008), 142-174. https://doi.org/10.1177/1555412008314132

[46] Teppo Räisänen, Antonia Ypsilanti, Don Ropes, Ana B Vivas, Matti Viitala, and Tuula Ijäs. 2014. Examining the requirements for an intergenerational learning game. Education and Information Technologies 19, 3 (2014), 531-547. https://doi.org/10.1007/s10639-014-9324-x

[47] Mark Rice, Yian Ling Cheong, Jamie Ng, Puay Hoe Chua, and Yin-Leng Theng. 2012. Co-creating games through intergenerational design workshops. In Proceedings of the designing interactive systems conference. ACM, NY, USA, 368-377. https://doi.org/10.1145/2317956.2318012

[48] Donald Ropes. 2013. Intergenerational learning in organizations. European fournal of Training and Development 37, 8 (2013), 713-727. https://doi.org/10.1108/EJTD-11-2012-0081

[49] Juan Pedro Serrano, Jose Miguel Latorre, Margaret Gatz, and Juan Montanes. 2004. Life review therapy using autobiographical retrieval practice for older adults with depressive symptomatology. Psychology and aging 19, 2 (2004), 272. https://doi.org/10.1037/0882-7974.19.2.272 
[50] Sinem Siyahhan and Elisabeth Gee. 2018. Families at Play: Connecting and Learning through Video Games. MIT Press, Cambridge, MA, USA.

[51] Dustin J Souders, Walter R Boot, Neil Charness, and Jerad H Moxley. 2016. Older adult video game preferences in practice: investigating the effects of competing or cooperating. Games and culture 11, 1-2 (2016), 170-200. https: //doi.org/10.1177/1555412015603538

[52] David Unruh. 1989. Toward a social psychology of reminiscence. Current Perspective on Aging and the Life Cycle 3 (1989), 25-46.

[53] Gerben J Westerhof and Ernst T Bohlmeijer. 2014. Celebrating fifty years of research and applications in reminiscence and life review: State of the art and new directions. fournal of Aging studies 29 (2014), 107-114. https://doi.org/10. 1016/j.jaging.2014.02.003

[54] Bob Woods, Sara Portnoy, Donna Head, and Gemma Jones. 1992. Reminiscence and life review with persons with dementia: which way forward. Care giving in dementia 1 (1992), 137-161.

[55] Xuexin Xu, Jinhui Li, Tan Phat Pham, Charles T Salmon, and Yin-Leng Theng. 2016. Improving psychosocial well-being of older adults through exergaming: the moderation effects of intergenerational communication and age cohorts. Games for health journal 5, 6 (2016), 389-397. https://doi.org/10.1089/g4h.2016.0060

[56] Li Yang. 2018. Long fourney of Life. Mobile Game [ios]. Last played August 2019.

[57] Fan Zhang. 2018. Intergenerational play between young people and old family members: patterns, benefits, and challenges. In International Conference on Human Aspects of IT for the Aged Population. Springer, Cham, 581-593. https://doi.org/10.1007/978-3-319-92034-4_44

[58] Fan Zhang, Simone Hausknecht, Robyn Schell, and David Kaufman. 2016. Factors Affecting the Gaming Experience of Older Adults in Community and Senior Centres. In International Conference on Computer Supported Education. Springer, Cham, 464-475. https://doi.org/10.1007/978-3-319-63184-4_24

[59] Fan Zhang and David Kaufman. 2016. A review of intergenerational play for facilitating interactions and learning. Gerontechnology 14, 3 (2016), 127-138.

[60] Fan Zhang, David Kaufman, Robyn Schell, Glaucia Salgado, Erik Tiong Wee Seah, and Julija Jeremic. 2017. Situated learning through intergenerational play between older adults and undergraduates. International fournal of Educational Technology in Higher Education 14, 1 (2017), 16. https://doi.org/10.1186/s41239-017-0055-0

[61] Renee'A Zucchero. 2010. Share your experience and I'll lend you my ear: older adult outcomes of an intergenerational service-learning experience. Gerontology \& Geriatrics Education 31, 4 (2010), 383-402. https://doi.org/10.1080/02701960 2010.528275

Received January 2020; revised June 2020; accepted July 2020 